\title{
CONFIABILIDAD Y VALIDEZ DE CRITERIO DEL CUESTIONARIO DEL SENTIDO DE LA VIDA EN PACIENTES CHILENOS EN HEMODIÁLISIS CRÓNICA ${ }^{1}$
}

\section{RELIABILITY AND CRITERION VALIDITY OF THE MEANING IN LIFE SCALE IN CHILEAN CHRONIC HEMODIALYSIS PATIENTS}

\author{
CARMen LuZ Muñoz-MendozA * \\ Miguel YÁÑEZ-ALVARAdo ** \\ Cecilia Pinto-Santuber ${ }^{* * *}$ \\ Patricia Letelier-SanZ ${ }^{* * *}$ \\ Teresa Balboa-Seguel ${ }^{* * * *}$ \\ M. DOLORES FERnÁNDEZ-PASCUAL ${ }^{* * * * *}$ \\ ABILIO REIG-FERRER ${ }^{* * * * * *}$
}

\begin{abstract}
RESUMEN
Objetivo: Evaluar la confiabilidad y validez de criterio de la versión española del Cuestionario del Sentido de la Vida (Meaning in Life Scale, MiLS) en pacientes chilenos en hemodiálisis crónica. Material y método: Estudio descriptivo transversal, sobre una muestra consecutiva de 244 personas en hemodiálisis crónica, beneficiarios del Servicio de Salud de la Región de Nuuble. El cuestionario MiLS consta de 21 ítems y cuatro dimensiones: Propósito de Vida, Falta de Significado, Paz Interior y Beneficios de la Espiritualidad. Se aplicó estadística descriptiva y análisis correlacional. La consistencia interna se determinó mediante el $\alpha$ de Cronbach. Los datos fueron analizados con el software estadístico SPSS v. 23. Resultados: La consistencia interna del cuestionario fue de 0,82. La puntuación global normalizada fue de 6,3. La puntuación global del cuestionario al igual que sus dimensiones correlacionan significativamente con todas las subvariables de bienestar subjetivo $(\mathrm{p}<0,01)$. Conclusión: El Cuestionario del Sentido de la Vida ha mostrado ser un instrumento viable, fiable y presentar apropiada validez de criterio para evaluar el bienestar espiritual de las personas en hemodiálisis. La evaluación del bienestar espiritual puede ser de utilidad para la práctica clínica.
\end{abstract}

Palabras clave: Insuficiencia Renal; Diálisis Renal; Espiritualidad; Reproducibilidad de los Resultados; Calidad de Vida; Atención de Enfermería.

\footnotetext{
*Enfermera, Doctora en Enfermería, Departamento de Enfermería, Facultad de Ciencias de la Salud y de los Alimentos, Universidad del Bío-Bío. Chillán, Chile.Email: calmunoz@ubiobio.cl. Autor de correspondencia.

** Licenciado en Matemática, Doctor en Estadística Multivariante Aplicada, Departamento de Estadística, Facultad de Ciencias, Universidad del Bío-Bío. Concepción, Chile. Email: myanez@ubiobio.cl

${ }^{* * *}$ Enfermera, Magíster en Pedagogía para la Educación Superior, Departamento de Enfermería, Facultad de Ciencias de la Salud y de los Alimentos, Universidad del Bío-Bío. Chillán, Chile. Email: cepinto@ubiobio.cl

${ }^{* * * *}$ Enfermera, Magíster en Enfermería, Departamento de Enfermería, Facultad de Ciencias de la Salud y de los Alimentos, Universidad del Bío-Bío. Chillán, Chile.Email: pletelie@ubiobio.cl

${ }^{* * * * *}$ Enfermera, Magíster en Administración Hospitalaria, Unidad de Diálisis, Centro de Responsabilidad Apoyo Terapéutico y Diagnóstico, Hospital Clínico Herminda Martín. Chillán, Chile. Email: teresa.balboa@redsalud.gov.cl

${ }^{* * * * * *}$ Psicóloga, Doctora en Psicología, Departamento de Psicología de la Salud, Facultad de Ciencias de la Salud, Universidad de Alicante. Alicante, España. Email: mariadolores.fernandezpascual@gmail.com

${ }^{* * * * * * * *}$ Psicólogo, Doctor en Psicología, Departamento de Psicología de la Salud, Facultad de Ciencias de la Salud, Universidad de Alicante. Alicante, España. Email: areig@ua.es
} 


\begin{abstract}
Objective: To evaluate the reliability and criterion validity of the Spanish version of the Meaning in Life Scale (MiLS) in Chilean chronic hemodialysis patients. Materials and methods: Cross-sectional descriptive study on a consecutive sample of 244 patients under chronic hemodialysis in the Nuble Region Health Service. The MiLS questionnaire consists of 21 items and 4 dimensions: Life Purpose, Lack of Meaning, Inner Peace and Benefits of Spirituality. Descriptive statistics and correlational analysis were applied. Internal consistency was determined using Cronbach's $\alpha$. Data were analyzed with the statistical software SPSS v. 23. Results: Internal consistency of the questionnaire was 0.82 . Standard global score was 6.3. The overall questionnaire score as well as the dimensions correlate significantly with all subjective well-being variables $(\mathrm{p}<0.01)$. Conclusions: The Meaning in Life Questionnaire has been shown to be a viable, reliable instrument and to present appropriate criterion validity to evaluate the spiritual well-being of patients under hemodialysis therapy. Consequently, the evaluation of spiritual well-being may be useful for clinical practice.
\end{abstract}

Key words: Renal insufficiency, renal dialysis, spirituality, reproducibility of results; quality of life; nursing care.

Fecha recepción: 14/06/18 Fecha aceptación: 04/10/18

\section{INTRODUCCIÓN}

Aunque los tratamientos actualmente disponibles para la Enfermedad Renal Crónica (ERC) en etapa terminal ayudan a sustituir en parte la función renal, a paliar sus síntomas y a preservar la vida de las personas, no proporcionan necesariamente una buena calidad de vida ${ }^{(1,2)}$.

La adaptación emocional que requieren las personas al someterse a sesiones periódicas de hemodiálisis puede ser una tarea difícil y que requiere de un proceso complicado, debido a los múltiples cambios que deben llevar a cabo: tiempo que debe dedicar a la diálisis, modificaciones en la dieta, restricciones en la ingesta de líquidos, entre otros. Frecuentemente, estos cambios repercuten no sólo en el funcionamiento físico, psicológico y social de la persona, sino que también la obligan a plantearse cuestiones de tipo espiritual ${ }^{(3)}$, es así como Puchalski et al. recomiendan prestar mayor atención a la espiritualidad en el cuidado ${ }^{(4)}$. Sin embargo, en la literatura existe una falta de consenso sobre la definición de espiritualidad ${ }^{(5)}$, existiendo algunos elementos comunes a todas las definiciones, que incluyen: la singularidad de la espiritualidad de cada persona; que es un constructo más amplio que las creencias religiosas o las afiliaciones; que es multidimensional; que hay una dimensión trascendental, que implica la conexión con uno mismo, otros, la naturaleza y/o un poder superior; y que está relacionado con la necesidad de encontrar un sentido a la vida ${ }^{(6)}$.

Numerosos estudios han informado que altos niveles de bienestar espiritual se relacionan significativamente con una mejor calidad de vida, adherencia con el tratamiento y una tasa más alta de supervivencia ${ }^{(7-9)}$.

En la literatura empírica internacional sobre espiritualidad existen numerosos instrumentos diseñados para medirla. En una revisión sistemática se identificaron 59 instrumentos, la mitad de los cuales evalúan sólo un aspecto del constructo o lo hacen dentro del contexto exclusivo de acontecimientos de vida negativos, aportando escasas evidencias sobre su calidad psicométrica ${ }^{(10)}$. Dentro de los instrumentos analizados destaca el Cuestionario del Sentido de la Vida ( $\mathrm{Me}$ aning in Life Scale, MiLS) ${ }^{(10)}$. Este cuestionario ha sido desarrollado y validado teniendo presente los modelos teóricos del sentido de la vida y de la espiritualidad de tipo existencial, donde el sentido de vida está presente cuando la persona tiene un propósito, coherencia, realización personal, y la creencia de que la vida tiene valor ${ }^{(11)}$. 
Las cuatro dimensiones del MiLS: Propósito de Vida, Falta de Significado, Paz Interior y Beneficios de la Espiritualidad, son consistentes con los modelos teóricos rigurosos e incorporan las áreas de trascendencia hacia uno mismo y más allá de uno mismo ${ }^{(11)}$.

En España, investigadores han realizado la adaptación del Cuestionario MiLS al español, en una muestra de 94 pacientes en hemodiálisis. Sus resultados evidenciaron que la versión española de este cuestionario es una medida de bienestar espiritual con garantías psicométricas de calidad, adecuada para evaluar las complejas exigencias generadas por la problemática de salud del paciente en hemodiálisis ${ }^{(12)}$.

En Chile no se dispone de un instrumento de medida de bienestar espiritual con garantías métricas de calidad: confiabilidad y validez. Por tanto, el objetivo principal de este estudio es evaluar la confiabilidad y la validez de criterio de la versión española del MiLS en personas chilenas en hemodiálisis crónica, mediante la confiabilidad por consistencia interna y la correlación con medidas de bienestar subjetivo y bienestar religioso.

\section{MATERIAL Y MÉTODO}

Estudio descriptivo transversal. De acuerdo a las técnicas psicométricas multivariantes, se recomiendan 10 personas por cada ítem del cuestionario ${ }^{(13)}$. Por tanto, se incluyeron 250 personas en hemodiálisis crónica, beneficiarios del Servicio de Salud de la Región de Nuble, de 18 y más años de edad, sin problemas de comprensión que les impidiera contestar los cuestionarios. Para este criterio, se excluyó a las personas con deficiencia cognitiva severa consignada en la ficha clínica. El profesional de enfermería de la Unidad o Centro de Diálisis, seleccionó mediante muestreo consecutivo a los participantes basándose en los criterios de elegibilidad.

Antes de comenzar el estudio, para garantizar la adecuación de sus aspectos metodológicos, éticos y jurídicos, el Comité Ético Científico del Hospital Clínico Herminda Martín de Chillán autorizó su ejecución. Además, a cada persona se explicó los objetivos de la investigación y se solicitó la firma del Consentimiento Informado.

Para la recogida de datos se utilizó una batería de preguntas. La primera parte estaba conformada por el Cuestionario MiLS, que consta de 21 ítems y 4 dimensiones ${ }^{(11,12)}$ :

1) Propósito de Vida: compuesta por 7 ítems, mide el grado de realización personal y satisfacción con la vida que la persona siente en la actualidad a partir de su situación personal;

2) Falta de Significado: formada por 7 ítems, indica una pérdida y disminución de valor y valía en la vida;

3) Paz Interior: constituida por 4 ítems, evalúa el grado de sensación interna de paz y armonía, de equilibrio personal en uno mismo;

4) Beneficios de la Espiritualidad: compuesta por 3 ítems, evalúa el grado de fuerza, fortaleza y consuelo que proporciona la fe religiosa $u$ otras creencias de tipo espiritual.

Las 4 dimensiones del cuestionario se responden con una escala tipo Likert: las dos primeras con una escala de seis alternativas de respuesta (desde muy en desacuerdo a muy de acuerdo) y las otras dos dimensiones con una de 5 posibilidades de respuesta (desde nada hasta mucho), basándose en el grado de verdad para cada una de las 7 afirmaciones que conforman los ítems 15 al 21. Además, de los valores para cada una de las dimensiones, el MiLS permite obtener una puntuación global de Espiritualidad. Esta puntuación total se obtiene a partir de las puntuaciones de los ítems en dirección positiva; para ello, se invierten tanto los valores de la escala Falta de Significado como del ítem 15. Al mismo tiempo, a los ítems con cinco alternativas de respuesta se les redefine con valores de 1 a $6(0=1,00 ; 1=2,25$; $2=3,50 ; 3=4,75$ y $4=6,00)$. A mayor puntuación en Espiritualidad total, mayor presencia de armonía personal y paz interior, mayor propósito y realización personal en la vida, mayor significado en la vida, y más beneficios percibidos ligados a las creencias de tipo espiritual. La versión española, para las cuatro dimensiones: Propósito de 
Vida, Falta de Significado, Paz Interior y Beneficios de la Espiritualidad, así como para la puntuación global, alcanzó valores de Alfa de Cronbach 0,$83 ; 0,80 ; 0,66 ; 0,90$ y 0,87 , respectivamente (12).

La segunda parte de la batería incluyó preguntas relativas a variables sociodemográficas (edad y sexo), clínica (tiempo en hemodiálisis), bienestar subjetivo (estado de salud general, calidad de vida general y felicidad personal) y bienestar religioso (grado de religiosidad y creencia en la existencia de una vida ultraterrena). Para evaluar el estado de salud general se utilizó la pregunta: "En general usted diría que su salud es:" (excelente, muy buena, buena, regular; mala). Para valorar calidad de vida general la pregunta: "Por lo general ¿diría que su calidad de vida es?” (muy buena, buena, regular, mala, muy mala). Para evaluar felicidad personal, la pregunta: "En líneas generales, diría que usted es muy feliz, bastante feliz, poco feliz o nada feliz". En el caso de la variable grado de religiosidad, se consultó: “ ¿Se considera usted una persona religiosa?” (escala de 4 alternativas desde muy religiosa a nada religiosa) y para creencia en la existencia de una vida ultraterrena: “¿Cree que existe algo después de la muerte?” (escala de 4 alternativas de respuesta desde nada hasta seguro que sí) ${ }^{(12)}$.

Los autores de la versión española del MiLS autorizaron el uso del cuestionario para su validación en nuestro contexto. Para evaluar la interpretabilidad de los ítems se realizó, previa a la administración definitiva, una prueba piloto con una muestra de 15 participantes, con características similares a los del estudio definitivo, y cuyos datos no fueron incluidos en el estudio final. Ningún ítem presentó problemas de comprensión por parte de los participantes.

La administración de la batería de preguntas se realizó en la Unidad o Centro de Diálisis por entrevistadores previamente capacitados, a objeto de disminuir los sesgos derivados de la aplicación del cuestionario.

Se aplicó estadística descriptiva y correlacional y de análisis diferencial en las variables de interés. En el análisis bivariado se aplicó el coeficiente de correlación de Spearman para esta- blecer la correlación con las variables sociodemográficas y clínica. Se estudió la consistencia interna del cuestionario a nivel global, y a nivel de cada una de las cuatro dimensiones utilizando el estadístico Alfa de Cronbach. Los datos obtenidos se analizaron con el programa estadístico SPSS v.23.

\section{RESULTADOS}

De las 250 personas seleccionadas se excluyeron $6(2,4 \%)$, al decidir suspender la entrevista. La muestra final quedó conformada por 244 personas, con una media de edad de 57,6 años (DE $15,6)$, y de los cuales el 54,5\% eran hombres. El $55,9 \%$ estaba casado y el 39,9\% tenía un nivel de enseñanza básica. El tiempo medio en hemodiálisis fue de 5,46 años (DE 4,9).

En la Tabla 1 se presentan las medias y desviaciones estándar, según reescalamiento y transformación propuestos en el estudio original, tanto para cada una de las dimensiones como para el Cuestionario MiLS global, los valores de los coeficientes Alfa de Cronbach y la normalización de las puntuaciones directas de cada una de las dimensiones y del cuestionario total en una escala de cero a diez. Como se puede observar, la consistencia interna global del cuestionario es muy buena $(0,82)$, mientras que en tres de las cuatro dimensiones los valores son satisfactorios; únicamente se obtiene un coeficiente más bajo en la dimensión Paz Interior $(0,57)$. En cuanto a la puntuación normalizada, 0 (peor puntuación) a 10 (mejor puntuación), las personas de la muestra obtienen una puntuación global de 6,3.

En la Tabla 2 se muestra el análisis correlacional entre las dimensiones del Cuestionario del Sentido de la Vida (MiLS) y las variables sociodemográficas, clínicas, de bienestar subjetivo y religioso. Como se puede observar, las dimensiones Propósito de Vida, Falta de Significado y Paz Interior muestran en términos absolutos correlaciones altas con la puntuación global del Cuestionario ( $r=0,73 ;-0,73$ y 0,71 , respectivamente). La relación entre la dimensión Falta de Signifi- 
Tabla 1. Análisis descriptivo y coeficientes de Alfa de Cronbach del Cuestionario del Sentido de la vida.

\begin{tabular}{lccc}
\hline & $\begin{array}{c}\text { Alfa de Cronbach } \\
(\alpha)\end{array}$ & $\begin{array}{c}\text { Puntuación } \\
\text { normalizada } \\
(0-10)\end{array}$ & Media (DE) \\
\hline Propósito de vida & 0,76 & 6,3 & $4,14(1,02)$ \\
Falta Significado & 0,78 & 4,4 & $3,19(1,13)$ \\
Paz Interior & 0,57 & 6,8 & $4,38(0,98)$ \\
Beneficios Espiritualidad & 0,84 & 7,5 & $4,77(1,33)$ \\
\hline Puntuación global Espiritualidad & 0,82 & 6,3 & $10,11(3,07)$ \\
\hline
\end{tabular}

Tabla 2. Análisis relacional entre las dimensiones del Cuestionario MiLS y con las variables de interés.

\begin{tabular}{|c|c|c|c|c|c|}
\hline & $\begin{array}{c}\text { Propósito } \\
\text { de vida }\end{array}$ & $\begin{array}{c}\text { Falta de } \\
\text { Significado }\end{array}$ & Paz Interior & $\begin{array}{c}\text { Beneficios } \\
\text { Espiritualidad }\end{array}$ & $\begin{array}{c}\text { Puntuación } \\
\text { Global }\end{array}$ \\
\hline \multicolumn{6}{|l|}{ Cuestionario MiLS: } \\
\hline Falta de Significado & $-0,18^{*}$ & & & & \\
\hline Paz Interior & $0,39^{*}$ & $-0,39^{*}$ & & & \\
\hline Beneficios Espiritualidad & $0,29^{*}$ & $-0,1$ & $0,46^{*}$ & & \\
\hline Puntuación Global & $0,73^{*}$ & $-0,73^{*}$ & $0,71^{\star}$ & $0,51^{\star}$ & \\
\hline \multicolumn{6}{|c|}{ Variables sociodemográficas: } \\
\hline Edad & 0,09 & $0,26^{\star}$ & $-0,01$ & 0 & $-0,11$ \\
\hline Sexo & $-0,03$ & 0,05 & 0,04 & $0,18^{\star}$ & 0,01 \\
\hline \multicolumn{6}{|l|}{ Variable clínica: } \\
\hline Tiempo HD $\dagger$ & 0 & 0,04 & 0,05 & 0,05 & 0,05 \\
\hline \multicolumn{6}{|c|}{ Variables Bienestar subjetivo: } \\
\hline Estado Salud & $0,34^{*}$ & $-0,35^{\star}$ & $0,31^{*}$ & $0,2^{*}$ & $0,4^{*}$ \\
\hline CV $\ddagger$ general & $0,4^{*}$ & $-0,21^{*}$ & $0,36^{*}$ & $0,17^{\star}$ & $0,4^{*}$ \\
\hline Felicidad personal & $0,35^{\star}$ & $-0,33^{*}$ & $0,41^{\star}$ & $0,26^{*}$ & $0,49^{\star}$ \\
\hline \multicolumn{6}{|c|}{ Variables Bienestar religioso: } \\
\hline Grado de Religiosidad & $0,18^{\star}$ & 0,04 & 0,12 & $0,34^{\star}$ & $0,23^{*}$ \\
\hline Vida ultraterrena & $0,19^{\star}$ & $-0,07$ & $0,24^{\star}$ & $0,44^{\star}$ & $0,36^{*}$ \\
\hline
\end{tabular}

${ }^{\star}$ Coeficientes de correlación de Spearman significativos ( $<<0,01$ ); †HD: Hemodiálisis; $¥$ CV: Calidad de Vida.

cado y la puntuación global resulta negativa, por lo que, al aumentar el puntaje de esta dimensión, influye en la disminución del puntaje total del cuestionario.
En cuanto a las variables sociodemográficas, el análisis muestra que la edad se relaciona significativamente $(p<0,01)$ con Falta de Significado $(r=0,26)$ y el sexo con Beneficios de la Espiri- 
tualidad $(r=0,18)$, ambas relaciones de carácter débil. La puntuación global del cuestionario al igual que todas sus dimensiones se correlacionan significativamente con todas las variables de bienestar subjetivo $(\mathrm{p}<0,01)$; a mayor grado de sentido de la vida dado por el puntaje global del cuestionario, mejor estado de salud, mayor calidad de vida general y mayor felicidad. Asimismo, la puntuación global se relaciona significativamente $(\mathrm{p}<0,01)$ con grado de religiosidad ( $\mathrm{r}$ $=0,23)$ y vida ultraterrena $(r=0,36)$. En cuanto a las dimensiones, Beneficios de la Espiritualidad es la que muestra correlaciones más altas con grado de religiosidad y vida ultraterrena, $r=0,34$ y $r=0,44$, respectivamente.

\section{DISCUSIÓN}

La espiritualidad es un aspecto esencial del ser humano ${ }^{(4,14,15)}$. Una revisión reciente señala que la discusión sobre temas espirituales en el ámbito sanitario es deseada por los pacientes, pero que en la práctica es poco común ${ }^{(16)}$. Existe una gran cantidad de evidencia empírica que recomienda la necesidad de incluir los cuidados espirituales dentro de una aproximación biopsicosocial y espiritual hacia los cuidados ${ }^{(4,17-19)}$, así como la importancia de investigar sobre las necesidades espirituales de los pacientes ${ }^{(20)}$. En este sentido, este estudio resulta relevante ya que por primera vez se dispone de un instrumento con propiedades métricas de calidad, en términos de confiabilidad y validez de criterio, que permite evaluar el grado de bienestar espiritual en pacientes chilenos en hemodiálisis crónica.

En relación a la viabilidad, aunque no fue evaluada directamente, el alto porcentaje de participantes (sólo un 2,4\% suspendieron la entrevista), permite establecer que fue muy buena, no representando una sobrecarga para el paciente o para el entrevistador. Aspecto similar a lo informado en el estudio de validación en pacientes españoles en hemodiálisis y en cuidados paliativos ${ }^{(12,21)}$.

En cuanto a los resultados psicométricos del cuestionario, la consistencia interna es satisfacto- ria, tanto para puntuación total como para tres de las dimensiones que lo conforman, solo la dimensión Paz Interior obtuvo un coeficiente más bajo. Estos resultados son consistentes con los del estudio original ${ }^{(11)}$ y los estudios de validación en población de habla hispana ${ }^{(12,21)}$. En cuanto al valor medio normalizado del Sentido de vida (0-10), en esta muestra fue de 6,3, superior al de la muestra española que alcanzó un valor de 5,1 . Esta diferencia puede deberse a que sólo un 9,9\% de los participantes del presente estudio se declara nada religioso en comparación con casi el $30 \%$ de la muestra española ${ }^{(12)}$. Lo mismo sucede con la puntuación normalizada de la dimensión Beneficios de la Espiritualidad del cuestionario, donde estos pacientes alcanzan una puntuación media de 7,5 frente al 4,4 de los pacientes españoles ${ }^{(12)}$.

El análisis correlacional de las dimensiones y de la puntuación global del cuestionario con las variables sociodemográficas, clínica, de bienestar subjetivo y religioso, tienden a evidenciar su validez de criterio: el bienestar espiritual se relaciona significativamente con las variables estado de salud, calidad de vida general y grado de religiosidad, y no muestra correlación con la edad, sexo y tiempo en hemodiálisis. Estos resultados coinciden con los de otras investigaciones ${ }^{(22-26)}$. El estudio de validación sobre una muestra de pacientes españoles en hemodiálisis encontró que la espiritualidad se asocia positiva y significativamente con variables de salud percibida, felicidad personal, calidad de vida y religiosidad, y presenta independencia del género, comorbilidad, edad y tiempo en hemodiálisis ${ }^{(12)}$. Resultados similares a los informados en estudios con muestras de pacientes renales crónicos (27-29) y sobre una muestra de pacientes de cuidados paliativos ${ }^{(21)}$. Una revisión sistemática sobre espiritualidad/religiosidad y Enfermedad Renal Crónica mostró que las creencias espirituales y religiosas se asocian significativamente con la carga de enfermedad, el malestar psicológico de tipo depresivo y una mejor calidad de vida ${ }^{(20)}$. En este sentido, los profesionales de enfermería pueden mejorar el bienestar espiritual y apoyar las necesidades espirituales de los pacientes para 
proporcionarles una atención integral y de cali$\operatorname{dad}^{(30,31)}$.

Aunque este estudio aporta importantes evidencias a la evaluación de la espiritualidad por medio del Cuestionario del Sentido de la Vida, no está exento de algunas consideraciones metodológicas: la técnica de muestreo no probabilística utilizada dificulta la generalización de los resultados. Sin embargo, el tamaño de la muestra y la elevada participación le otorga relevancia clínica a estos resultados. Por otro lado, si bien la validez de criterio se realizó con variables relevantes para el bienestar espiritual, no existen instrumentos considerados como gold standard aceptados en el área.

\section{CONCLUSIÓN}

La versión española del Cuestionario del Sentido de la Vida ha mostrado ser un instrumento con criterios psicométricos de calidad en términos de viabilidad clínica, fiabilidad y relacionarse adecuadamente con variables de bienestar subjetivo y bienestar religioso, medidas a través de preguntas. Asimismo, pone a disposición de los profesionales de la salud un instrumento multidimensional adecuado y útil para medir el impacto de las intervenciones sobre la calidad de vida relacionada con la salud de los pacientes, desde la perspectiva de la espiritualidad.

\section{REFERENCIAS}

1. Ducharlet K, Sundarajan V, Philip J, Weil J, Barker N N, Langham RG, Burchell J, Gock H. Patient-Reported outcome measures and their utility in the management of patients with advanced chronic kidney disease. Nephrology (Carlton) [Internet]. 2018 Oct 8. doi: 10.1111/nep.13509. [Epub ahead of print].

2. Rodríguez Vidal M, Merino Escobar M, Castro Salas M. Valoración psicométrica de los componentes físicos (CSF) y mentales (CSM) del SF-36 en pacientes insuficientes renales crónicos en tratamiento con hemodiálisis. Cienc. enferm. 2009; 15(1): 75-88.

3. Musa AS, Pevalin DJ, Al Khalaileh MAA. Spiritual Well-Being, Depression, and Stress Among Hemodialysis Patients in Jordan. J Holist Nurs [Internet]. 2018 Dec. [cited 2018 May 25]; 36(4): 354-365. Available from: https://www.ncbi.nlm.nih.gov/ pubmed/29173010

4. Puchalski C, Ferrell B, Virani R, Otis-Green S, Baird P, Bull J, et al. Improving the quality of spiritual care as a dimension of palliative care: the report of the Consensus Conference. J Palliat Med. 2009; 12(10): 885-904.

5. Koenig HG, Larson DB, Larson SS. Religion and coping with serious medical illness. Ann Pharmacother. 2001; 35(3): 352-9.

6. Weathers E, McCarthy G and Coffey A. Concept analysis of spirituality: an evolutionary approach. Nurs Forum [Internet]. 2016 Apr [cited 2018 May 25]; 51(2): 79-96. Available from: https://www.ncbi.nlm.nih.gov/ pubmed/25644366

7. Lucchetti G, De Almeida LG, Lucchetti AL. Religiousness, mental health, and quality of life in Brazilian dialysis patients. Hemodial Int [Internet]. 2012 Jan [cited 2018 May 25]; 16(1): 89-94. Available from: https://www. ncbi.nlm.nih.gov/pubmed/22099479

8. Tanyi RA, Werner JS. Women's experience of spirituality within end-stage renal disease and hemodialysis. Clin Nurs Res. 2008; 17(1): 32-49.

9. Spinale J, Cohen SD, Khetpal P, Peterson RA, Clougherty B, Puchalski CM, et al. Spirituality, social support, and survival in hemodialysis patients. Clin J Am Soc Nephrol. 2008; 3(6): 1620-1627.

10. Brandstätter $M$, Baumann U, Borasio GD, Fegg MJ. Systematic review of meaning in life assessment instruments. Psychooncology [Internet]. 2012 Oct [cited 2018 May 25]; 21(10): 1034-1052. Available from: https:// www.ncbi.nlm.nih.gov/pubmed/22232017

11. Jim HS, Purnell JQ, Richardson SA, Gold- 
en-Kreutz D, Andersen BL. Measuring meaning in life following cancer. Qual Life Res. 2006t; 15(8): 1355-71.

12. Reig-Ferrer A, Arenas MD, Ferrer-Cascales R, Fernández-Pascual MD, Albaladejo-Blázquez N, Gil MT, et al. Evaluación del bienestar espiritual en pacientes en hemodiálisis. Nefrología (Madr.) [Internet] 2012 Apr [cited 2017 Dec 14]; 32(6): 731-742. Disponible en: http://scielo.isciii.es/scielo.php?script $=$ sci_ arttext\&pid=S0211-69952012000800006\&l$\mathrm{ng}=\mathrm{es}$

13. Hair J, Anderson R, Tatham R, Black W. Análisis Multivariante. $5^{\text {ta }}$ ed. Madrid: Prentice Hall Iberia; 1999. 832 p.

14. McCord G, Gilchrist VJ, Grossman SD, King BD, McCormick KE, Oprandi AM et al. Discussing spirituality with patients: a rational and ethical approach. Ann Fam Med. 2004; 2(4): 356-61.

15. Balboni TA, Vanderweker LC, Block SD, Paulk ME, Lathan CS, Peet JR et al. Religiousness and spiritual support among advanced cancer patients and associations with end-oflife treatment preferences and quality of life. J Clin Oncol. 2007; 25(5): 555-60.

16. Best M, Butow P, Olver I. Doctors discussing religion and spirituality: A systematic literature review. Palliat Med [Internet]. 2016 Apr [cited 2018 May 25]; 30(4): 327-337. Available from: https://www.ncbi.nlm.nih.gov/ pubmed/26269325

17. Martins Castro MC. Reflections on end-oflife dialysis. J. Bras. Nefrol. [Internet]. 2018 Jul-Sept [cited 2018 May 25]; 40(3): 233-241. [Epub 2018 May 17]. Available from: http:// www.scielo.br/scielo.php?script=sci_arttext\&pid=S0101-28002018005010101

18. Davison SN, Jhangri GS. Existential and religious dimensions of spirituality and their relationship with health-related quality of life in chronic kidney disease. Clin J Am Soc Nephrol [Internet]. 2010 Nov [cited 2018 May 25]; 5(11): 1969-1976. Available from: https://www.ncbi.nlm.nih.gov/ pubmed/20651152

19. Davison SN, Jhangri GS. The relationship between spirituality, psychosocial adjustment to illness, and health-related quality of life in patients with advanced chronic kidney disease. J Pain Symptom Manage [Internet]. 2013 Feb [cited 2018 May 25]; 45(2): 170178. Available from: https://www.ncbi.nlm. nih.gov/pubmed/22917713

20. Bragazzi N, Del Puente G. Chronic Kidney Disease, Spirituality and Religiosity: A Systematic Overview with the List of Eligible Studies. Health Psychol Res [Internet]. 2013 Apr [cited 2018 May 25]; 1(2): 135-140. Available from: https://www.ncbi.nlm.nih. gov/pmc/articles/PMC4768585/

21. Reig-Ferrer A, Ferrer-Cascales R, Fernández-Pascual MD, Natalia Albaladejo-Blázquez N, Priego Valladares M. Evaluación del bienestar espiritual en pacientes en cuidados paliativos. Med Paliat [Internet]. 2015 AbrJun [citado 2018 May 25]; 22(2): 60-68. Disponible en: https://www.sciencedirect.com/ science/article/pii/S1134248X13000864

22. Fradelos EC, Tzavella F, Koukia E, Papathanasiou IV, Alikari V, Stathoulis J et al. Integrating chronic kidney disease patient's spirituality in their care: health benefits and research perspectives. Mater Sociomed [Internet]. 2015 Oct [cited 2018 May 25]; 27(5): 354-358. Available from: https://www.ncbi. nlm.nih.gov/pubmed/26622206

23. Martínez BB, Custódio RP. Relationship between mental health and spiritual wellbeing among hemodialysis patients: a correlation study. Sao Paulo Med. J. [Internet] 2014 [cited 2017 Dec 14]; 132(1): 23-27. Available from: https://www.ncbi.nlm.nih.gov/ pubmed/24474076

24. Lucchetti G, Camargo de Almeida LG, Lamas Granero AL. [Spirituality for dialysis patients: should the nephrologist address?] J. Bras. Nefrol. 2010; 32(1): 126-130.

25. Berman E, Merz JF, Rudnick M, Snyder RW, Rogers KK, Lee J et al. Religiosity in a hemodialysis population and its relationship to satisfaction with medical care, satisfaction with life, and adherence. Am J Kidney Dis [Internet]. 2004 Sept [cited 2018 May 25]; 
44(3): 488-497. Available from: https://www. ncbi.nlm.nih.gov/pubmed/15332222

26. Kimmel PL, Emont SL, Newmann JM, Danko H, Moss AH. ESRD patient quality of life: symptoms, spiritual beliefs, psychosocial factors, and ethnicity. Am J Kidney Dis. [Internet]. 2003 Oct [cited 2018 May 25]; 42(4): 713-721. Available from: https://www.ncbi. nlm.nih.gov/pubmed/14520621

27. Loureiro ACT, de Rezende Coelho MC, Coutinho FB, Borges LH, Lucchetti G.The influence of spirituality and religiousness on suicide risk and mental health of patients undergoing hemodialysis. Compr Psychiatry [Internet]. 2018 Jan [cited 2018 May 25]; 80: 39-45. Available from: https://www.ncbi.nlm. nih.gov/pubmed/28972917

28. Cruz JP, Colet PC, Alquwez N, Inocian EP, Al-Otaibi RS, Islam SM. Influence of religiosity and spiritual coping on health-related quality of life in Saudi haemodialysis patients. Hemodial Int [Internet]. 2017 Jan [cited 2018 May 25]; 21(1): 125-132. Available from: https://www.ncbi.nlm.nih.gov/ pubmed/27329681
29. Ottaviani AC, Souza ÉN, Drago N de C, de Mendiondo MS, Pavarini SC, Orlandi F de S. Hope and spirituality among patients with chronic kidney disease undergoing hemodialysis: a correlational study. Rev Lat Am Enfermagem. [Internet]. 2014 Mar-Apr [cited 2017 Dec 14]; 22(2): 248-254. Available from: https://www.ncbi.nlm.nih.gov/ pubmed/26107832

30. Egan R, Wood S, MacLeod R, Walker R. Spirituality in Renal Supportive Care: A Thematic Review. Healthcare (Basel) [Internet]. 2015 Nov [cited 2018 May 25]; 16; 3(4): 11741193. Available from: https://www.ncbi.nlm. nih.gov/pubmed/27417819

31. Vitorino LM, Soares RCES, Santos AEO, Lucchetti ALG, Cruz JP, Cortez PJO et al. Two Sides of the Same Coin: The Positive and Negative Impact of Spiritual Religious Coping on Quality of Life and Depression in Dialysis Patients. J Holist Nurs [Internet]. 2018 Dec [cited 2018 May 25]; 36(4): 332-340. [Epub 2017 Aug 24]. Available from: https:// www.ncbi.nlm.nih.gov/pubmed/28836475 